\title{
Knee joint preservation surgery in osteosarcoma using tumour-bearing bone treated with liquid nitrogen
}

\author{
Takashi Higuchi $^{1}$ - Norio Yamamoto ${ }^{1}$ Hideji Nishida ${ }^{1}$ - Katsuhiro Hayashi ${ }^{1}$. \\ Akihiko Takeuchi ${ }^{1}$ - Hiroaki Kimura ${ }^{1}$ - Shinji Miwa ${ }^{1}$ - Hiroyuki Inatani ${ }^{1}$. \\ Shingo Shimozaki ${ }^{1}$. Takashi Kato ${ }^{1}$ - Yu Aoki ${ }^{1}$ Kensaku Abe ${ }^{1}$. Yuta Taniguchi ${ }^{1}$. \\ Hiroyuki Tsuchiya ${ }^{1}$
}

Received: 28 December 2016 / Accepted: 24 April 2017 /Published online: 1 June 2017

(C) The Author(s) 2017. This article is an open access publication

\begin{abstract}
Purpose To preserve the joint structure in order to maintain good limb function in patients with osteosarcoma, we perform epiphyseal or metaphyseal osteotomy and reconstruction using frozen autografts that contain a tumour treated with liquid nitrogen. There are two methods of using liquid nitrogen-treated autografts: the free-freezing method and the pedicle-freezing method. The purpose of this study was to evaluate the results of intentional joint-preserving reconstruction using the free-freezing method and the pedicle-freezing method in patients with osteosarcoma.

Methods Between 2006 and 2014, we performed jointpreserving surgery (12 with the free-freezing method and six with the pedicle freezing method) to treat 18 cases of osteosarcoma (12 distal femurs and six proximal tibias) in patients who had achieved a good response to neoadjuvant chemotherapy.

Results Among the 18 patients (nine boys and nine girls) who had a mean age of 11.6 years, 13 remained continuously disease-free, three showed no evidence of disease, one was alive with the disease, and one died from the disease. Functional outcomes were assessed as excellent in 15 patients and poor in three, with a mean follow-up period of 46.1 months. The mean Musculoskeletal Tumour Society (MSTS) score was 90.2\%. Except for one patient who underwent amputation, all patients could bend their knee through $>90^{\circ}$ flexion, and nine achieved full ROM. All but two patients could walk without aid, and 11 were able to run normally throughout the follow-up period.
\end{abstract}

Hiroyuki Tsuchiya

tsuchi@med.kanazawa-u.ac.jp

1 Department of Orthopaedic Surgery, Graduate School of Medical Science, Kanazawa University, 13-1 Takara-machi,

Kanazawa 920-8641, Japan
No intraoperative complications were observed, such as surrounding soft-tissue damage, neurovascular injury, or recurrence from frozen bone.

Conclusions Joint-preserving reconstruction using frozen autografts yielded excellent function in patients with osteosarcoma.

Keywords Osteosarcoma $\cdot$ Knee joint $\cdot$ Liquid nitrogen · Frozen autografts $\cdot$ Joint-preserving reconstruction $\cdot$ Function

\section{Introduction}

According to Manfrini et al., saving the knee joint is the main goal in this type of limb salvage, in the belief that no joint replacement, either prosthetic or allograft, functions better than an intact knee [1]. Wong et al. also reported that in the resection of primary bone sarcomas, sparing the articular end of the affected bone enables patients to retain their native joints and ligaments [2]. This approach may result in better proprioception and more normal joint function after reconstruction. The purpose of a wide surgical margin is to reduce the risk of local recurrence. However, additional resection of normal tissue can jeopardize vital structures, including ligaments, tendons, and physes, and can possibly lead to restricted limb function and limb length discrepancy [3]. The traditional 2-3-cm-wide margin has recently been questioned, and methods using a reduced margin and partial epiphyseal preservation to improve host tissue preservation have been reported [3-6]. In these studies, patients have experienced better functional results and acceptable local disease control.

Previously, we reported performing transepiphyseal osteotomy and resecting a malignant bone tumour followed by reconstruction using distraction osteogenesis [7]. We subsequently developed a new biological reconstruction method 
with a massive frozen tumour-bearing bone treated with liquid nitrogen $[8,9]$. The development of the liquid nitrogen method was based on in vitro and in vivo studies of the hypothermic effects of liquid nitrogen. On basic study, we confirmed that only one cycle of $-196{ }^{\circ} \mathrm{C}$ for $20 \mathrm{~min}$ is sufficient to kill all tumour cells by inducing ice crystal formation and cell dehydration. Since then, we have clinically applied the liquid nitrogen method to musculoskeletal tumour surgery [9].

In the present study, we combined these techniques: we performed transepiphyseal osteotomies and resected tumours followed by the reconstruction of massive frozen tumourbearing bones treated with liquid nitrogen for joint preservation. We assessed clinical and functional outcomes, complications, and bone union time.

\section{Patients and methods}

From 2006 to 2014, 18 patients with osteosarcoma (nine boys and nine girls; mean age, 11.6 years) underwent jointpreserving tumour resection (Table 1). Tumours were located in the distal femur in 12 patients and in the proximal tibia in six. The minimum follow-up period was two years (mean, 46.1 months; range, 24-79 months). The base protocol of chemotherapy was given as five pre-operative courses of intra-arterial or intra-venous cisplatin $\left(120 \mathrm{mg} / \mathrm{m}^{2}\right)$ and doxorubicin $\left(30 \mathrm{mg} / \mathrm{m}^{2} /\right.$ day $\times 2$ days), according to our chemotherapy regimen [10]. Before surgery, chemotherapeutic effects were clinically evaluated after three to five courses of preoperative chemotherapy. Evidence of a good chemotherapeutic response included sclerotic changes or good delineation of the tumour (observed on plain radiographs), marked shrinkage of any extension of the tumour into soft tissue (demonstrated on MRI), or a decrease in the abnormal accumulation of ${ }^{201} \mathrm{Tl}$ on scintigrams.

The surgical margin was planned to make wide margin with the preservation of joint subchondral bone on the basis of the MRI T1 sequence and gadolinium enhancement analyses. During the operation, transepiphyseal osteotomy was important for providing an adequate margin. A K-wire was inserted into the osteotomy line under fluoroscopy. The tumour was then excised en bloc using a microsurgical saw. The specimen's soft tissue was removed, and the tumour was curetted before freezing. The excised portion was frozen in liquid nitrogen that was stored in sterilized flask right before freezing for 20 minutes, thawed at room temperature for $15 \mathrm{mi}-$ nutes, thawed in distilled water for 15 minutes, and then replaced with locking plates and screws. Cancellous allografts or artificial bone grafts were used for the defect if the defect became large. This technique is referred to as the "free-freezing method" (Fig. 1a). These methods were performed in 11 patients. To avoid non-union of the host-graft junction in the free-freezing method, a new surgical technique, called the "pedicle-freezing method," was developed [11]. The tumours were exposed and separated from the surrounding soft tissue with adequate safe surgical margins. Then, after osteotomy at the proximal site of the tumour, more soft tissue was divided with a safe surgical margin to prevent damage to normal soft tissue during liquid nitrogen treatment. A tourniquet was applied to the affected limb to prevent bleeding during curettage and to prevent the distant dissemination of tumour cells. The base of the elevated bone was isolated using cotton for cast padding, an Esmarch bandage, and a few surgical sheets to prevent tumour contamination and damage to the normal tissue when freezing. The bony lesion was then cautiously rotated down into the liquid nitrogen. After freezing, reconstruction was performed using locking plates and screws (Fig. 1b). Preferred sites for the pedicle freezing method are proximal tibia, proximal femur, and proximal humerus. Shaft to distal femur is also an indicated site for this method. However, in this case, the tumour location should be at least $5 \mathrm{~cm}$ distant to the structures to be preserved (e.g., articular cartilage or epiphyseal cartilage) for avoiding the risk of liquid nitrogen infiltration and for plate fixation of the junction between the frozen bone and the preserved structures. In the present study, the pedicle-freezing method was performed in five proximal femurs and one shaft to distal femur.

An additional six courses of post-operative chemotherapy were administered from two to three weeks after the operation [10]. All patients waited for weight-bearing (usually for 23 months) until initial bone union was detected by radiography.

Indications for the treatment of joint-preserving tumour resections using the free-freezing and pedicle-freezing methods included joints with an articular surface, subchondral bone with a collateral metaphyseal cortex preserved after adequate excision of the tumour, and joints in which internal fixation with plates and screws is possible.

Post-operative functional results were evaluated at the final follow-up using the Musculoskeletal Tumour Society (MSTS) score [12]. In addition, oncologic outcome, bone union time (when the continuity of the bone cortex at the host-graft junction was observed using plural directions radiography, tomosynthesis imaging, or CT scans), active range of motion (ROM) of the knee, and walking activity were recorded.

This was a retrospective clinical review study and was approved by the local institutional review board. All subjects were informed that data from this study would be submitted for publication and gave their consent for participation.

\section{Results}

At the latest follow-up, 13 patients were continuously diseasefree. Three had local recurrences from the surrounding soft tissue and two had distant metastases. There was no 


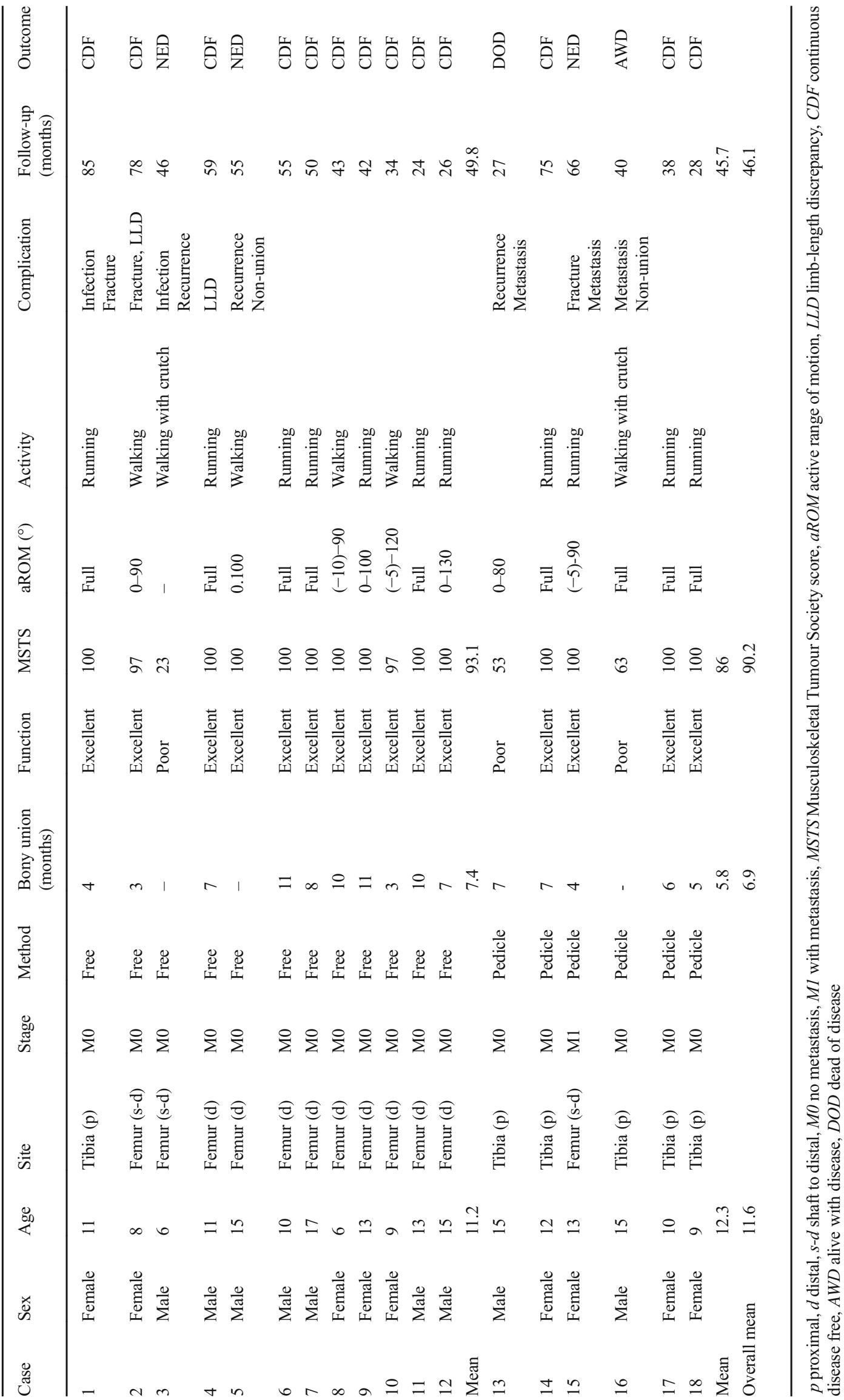


Fig. 1 a Intercalary osteotomy and free-freezing method.

Tumour of the distal femur is excised by transepiphyseal and intercalary osteotomy. After freezing in liquid nitrogen, osteosynthesis using plates, screws, and bone graft at the hostgraft junction is performed. b One-site osteotomy and pediclefreezing method. Tumour of the proximal tibia is excised by onesite osteotomy and curettage of tumour. After pedicle freezing in liquid nitrogen, osteosynthesis using plate, screws, and bone graft at the host-graft junction is performed

\section{a}
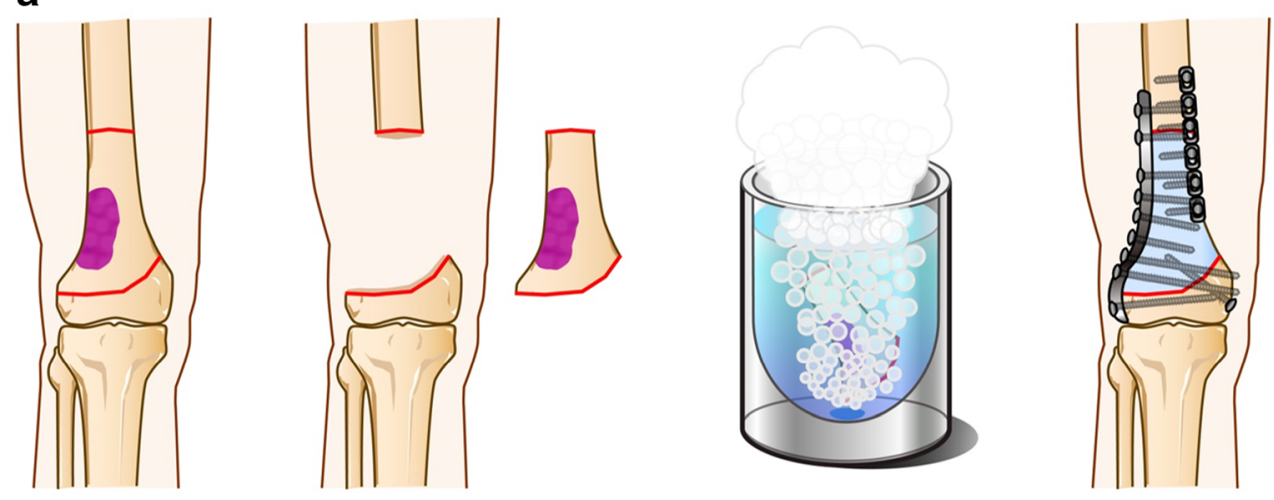

b

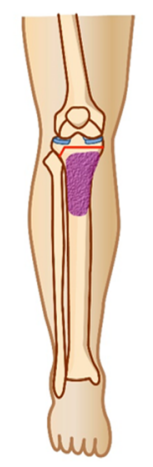

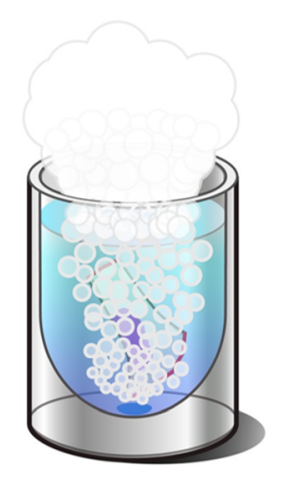

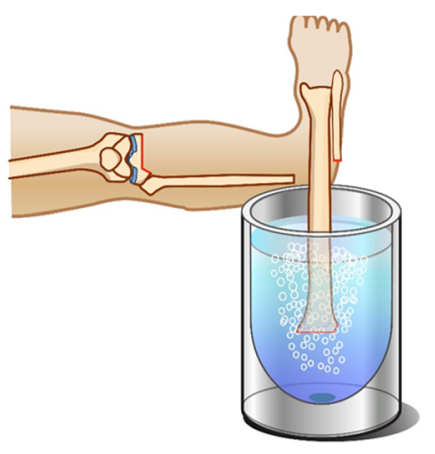

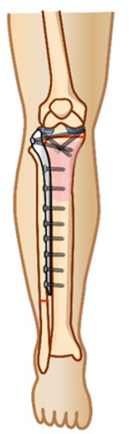

recurrence from the frozen bone. The two patients with metastases underwent metastasectomy, and the three patients with local soft tissue recurrences underwent additional chemotherapy and surgery (one underwent wide excision alone, one underwent wide excision and osteoarticular pedicle freezing because the recurrent tumour was adjacent to the remaining joint ligament and preserved epiphyseal bone, and the third, with a coexisting deep infection, was amputated). Ultimately, three of these five patients showed no evidence of the disease, one was alive with the disease, and one died from the disease.

Post-operative complications such as fracture (three) or non-union (two) were managed by revision osteosynthesis. Infection was seen in two patients (one had a deep infection and the other had a superficial infection). Both cases with infection were treated by the free-freezing method, and no infection was detected in the patients treated by the pediclefreezing method. No other complications were observed, including intra-operative fracture or neurovascular injury. Amputation was performed in one patient because of deep infection and local recurrence from the surrounding soft tissue.

The mean time to complete bone union was 6.9 months (range, 3-11 months). There was a tendency to shorter bone union time in the patients with pedicle-freezing (5.8 months vs. 7.4 months). Post-operative function was excellent in 15 patients and poor in three. The mean MSTS score was 90.2\% (range, 23-100\%). Except for the patient who underwent amputation, all patients could bend their knee through $>90^{\circ}$ flexion and 50\% (five patients with proximal tibial lesions and four with distal femur lesions) achieved full ROM during the follow-up period. All but two patients could walk without aid, and 11 were able to run normally at the latest follow-up (Table 1).

\section{Case presentations}

Case 14 (pedicle-freezing method): A 12-year-old girl presented with osteosarcoma of the proximal tibia. Neoadjuvant chemotherapy was administered, and marked shrinkage and ossification of the tumour were observed. MRI showed a metaphyseal tumour localized in the proximal tibia. We planned the pedicle-freezing method. After transepiphyseal osteotomy, the soft tissue was divided along the extremity. Bony lesions connected to the limb were then rotated and frozen in liquid nitrogen (Fig. 2). Reconstructions were performed using a plate and cannulated screws. The resection margins were tumour-free, which was pathologically confirmed by the small sample collected from preserved host tissue. Pathological evaluation from the curettage cancellous bone indicated total necrosis of the tumour, classified as grade IV/IV in the Rosen and Huvos evaluation system [13]. After 
Fig. 2 Case 14: A 12-year-old girl with an osteosarcoma of the proximal tibia. a Pre-operative MRI scan. b Osteotomy along a $\mathrm{K}$-wire was performed under fluoroscopy. c After transepiphyseal osteotomy and exposed tumour-bearing bone. $\mathbf{d}$ The bony lesion connecting with the limb was rotated and frozen in liquid nitrogen. e After freezing [30]
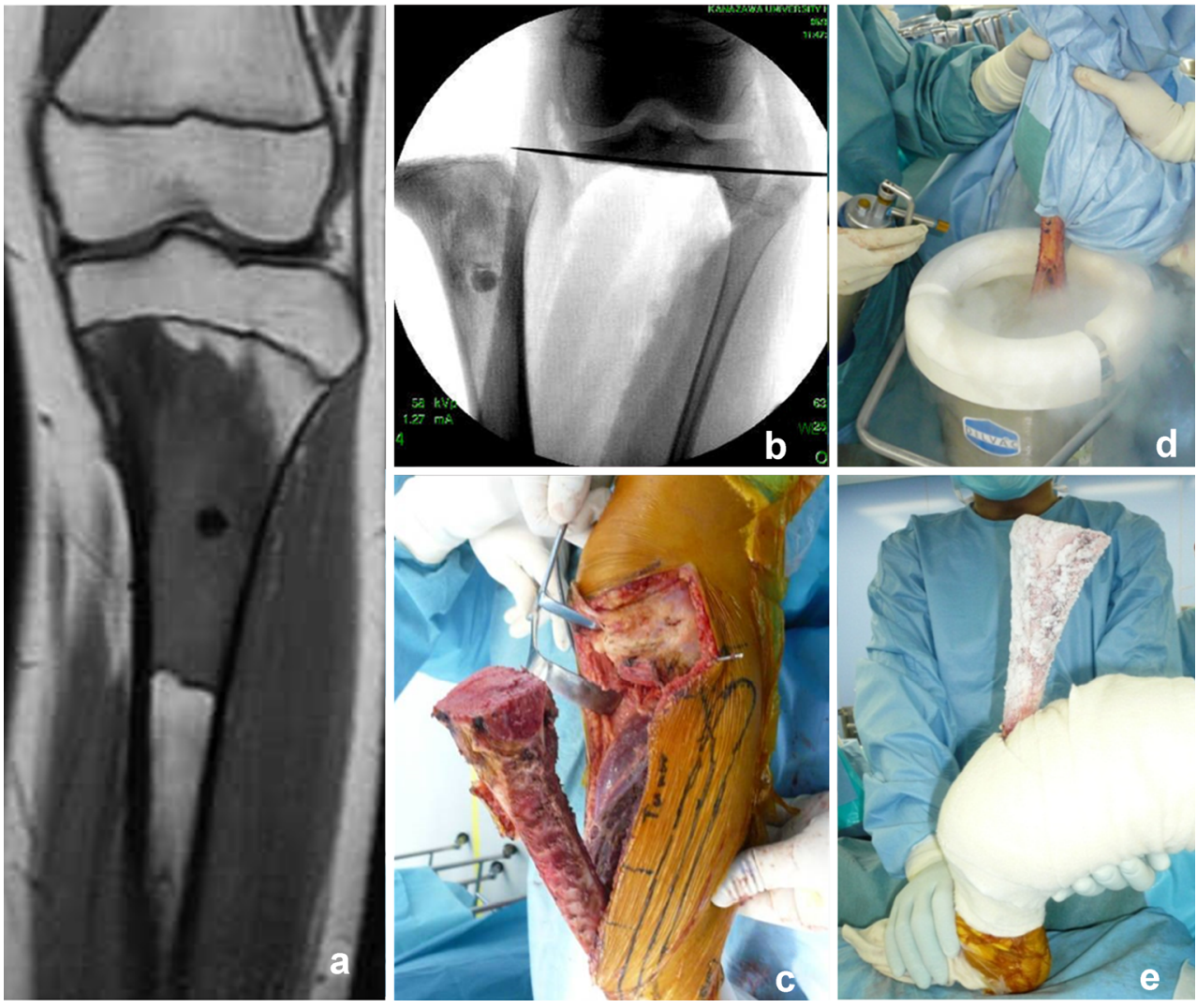

the completion of post-operative chemotherapy, the patient was disease-free and could run normally with full knee ROM at her 75-month follow-up (Fig. 3).

Case 4 (free-freezing method): An 11-year-old boy presented with osteosarcoma of the distal femur. Neoadjuvant chemotherapy was administered, and the clinical effect was a complete response with marked shrinkage and ossification of the tumour. We performed transepiphyseal and intercalary resection and reconstruction using the free-freezing method (Fig. 4). Satisfactory bone union was found seven months

Fig. 3 Case 14. a Osteosynthesis with plate, screws, and patella tendon fixed with spike washer. b Radiograph after reconstruction. c Seventy-eight months after reconstruction, no extension lag and full range of motion
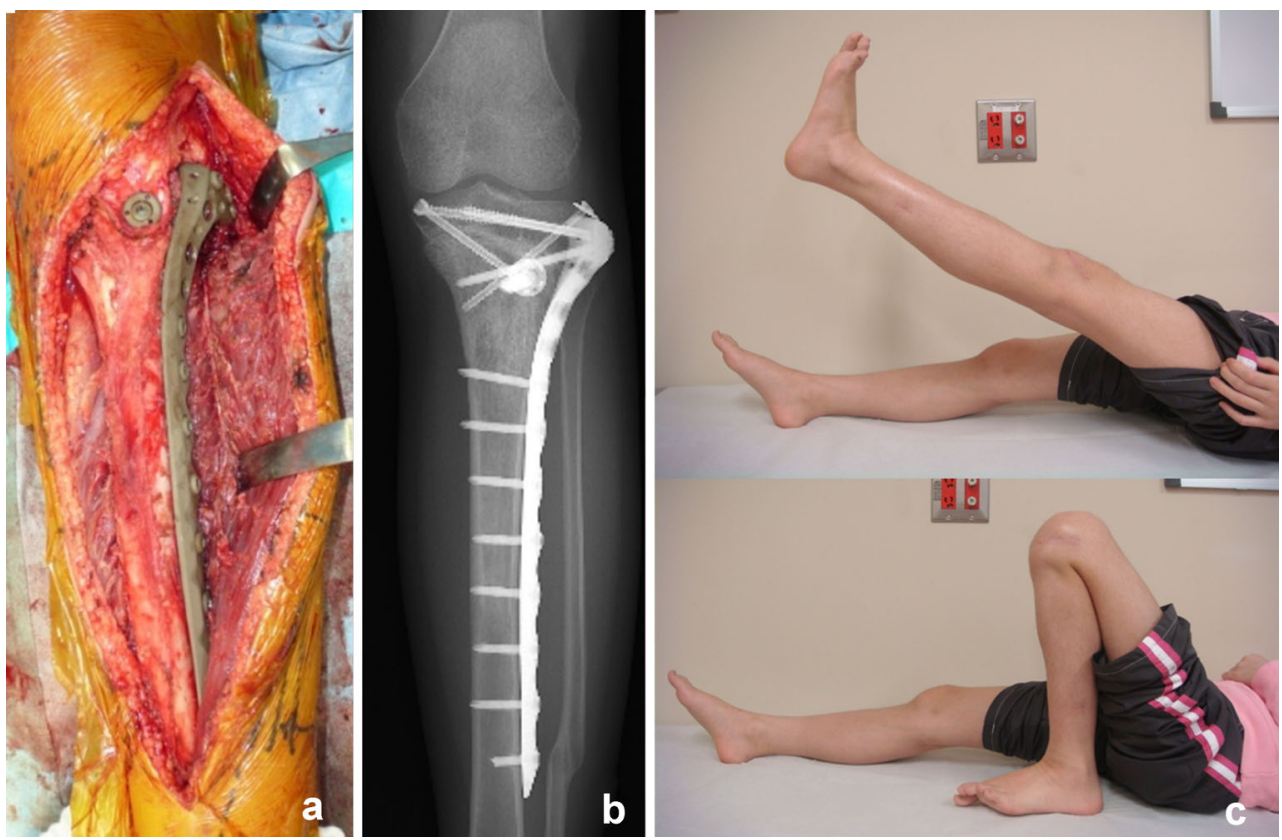
Fig. 4 Case 4: An 11-year-old boy with an osteosarcoma of the distal femur. a Pre-operative MRI scan. Osteotomy line (dotted line). b A K-wire was inserted into the osteotomy line under fluoroscopy. c The femur after transepiphyseal and intercalary osteotomy. d Tumour bearing bone. e Freezing in the liquid nitrogen. $\mathbf{f}$ After freezing. After transepiphyseal osteotomy and exposed tumour-bearing bone. $\mathbf{g}$ Osteosynthesis with plates, screws, and wire

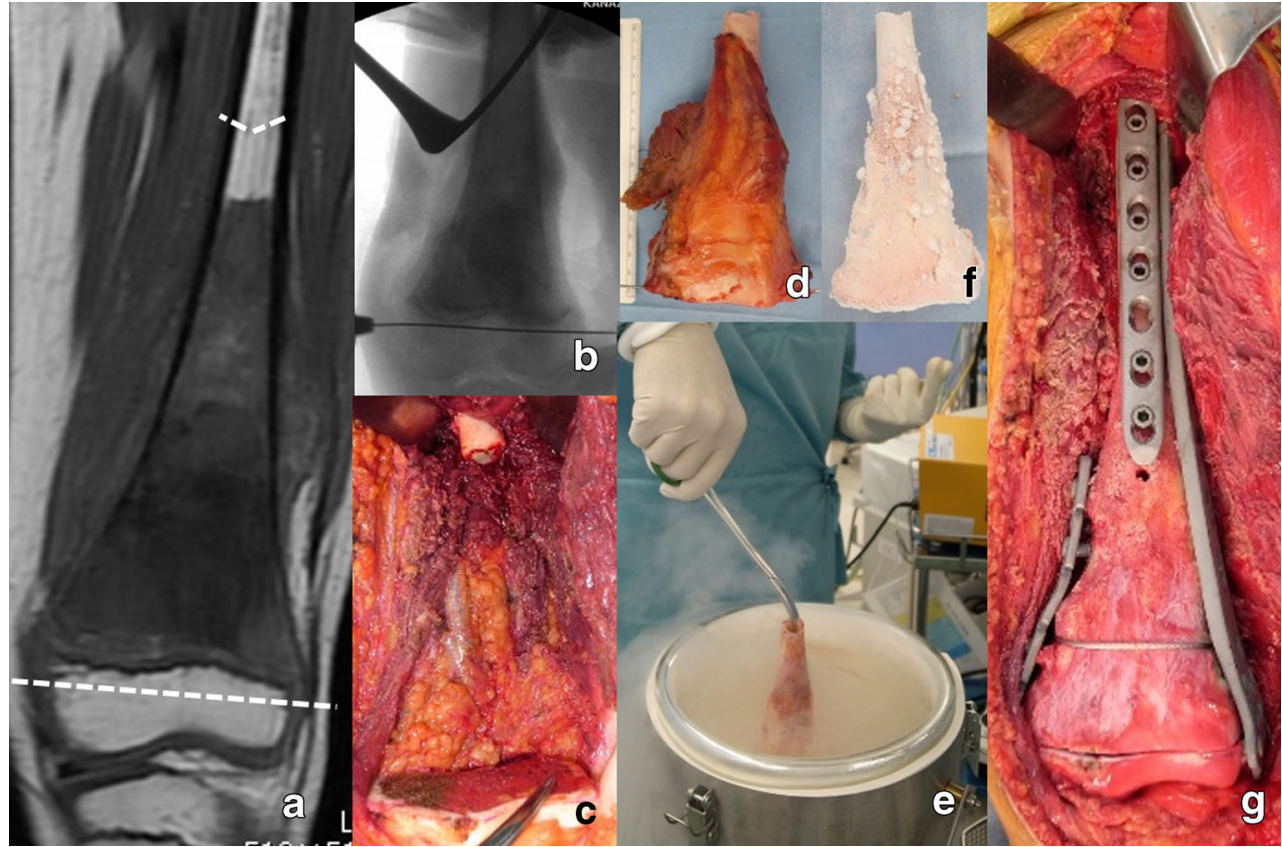

after surgery; however, at 3.5 years after the surgery, limblength discrepancy became a problem, and limb lengthening was performed with external fixation (Fig. 5). At his 43-month follow-up, the patient was disease-free and was able to play football.

\section{Discussion}

Recent advances in multimodality therapy have resulted in limb-sparing surgery being standard for high-grade osteosarcomas. Furthermore, for selected patients, joint-sparing

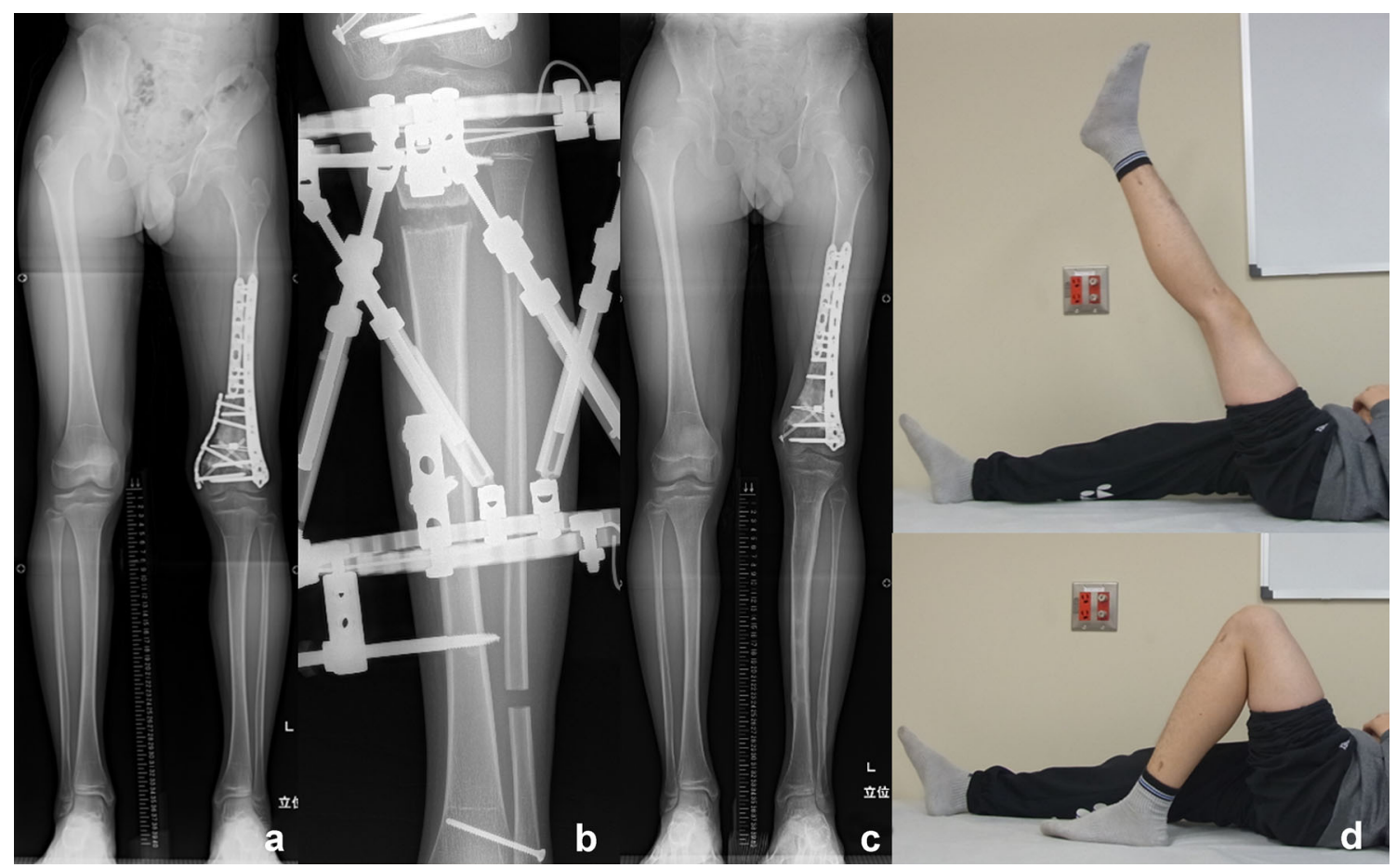

Fig. 5 Case 4. a After 3.5 years. Limb-length discrepancy. b Limb lengthening with external fixation. c After lengthening. Limb-length discrepancy has been improved. d At his 43-month follow-up, the patient was disease-free and was able to play football with no extension lag and full range of motion of the knee 
surgery is possible, allowing preservation of the joint structure in an effort to maintain normal limb function and avoid complications associated with endoprostheses.

Recently, many reports have confirmed that a custom diaphyseal implant (CDI) allows filling of the gap after jointsparing diaphyseal resection and enables immediate weightbearing and good knee function preservation [14]. Furthermore, when the growth plate is sacrificed, the option of expansion in some CDIs will prevent limb discrepancy. However, the durability of artificial materials could be a problem in the long term, requiring revision surgery due to loosening or damaged prostheses. The reconstruction of soft tissue attachments is also a major problem because artificial materials do not biointegrate into soft tissues, such as muscles and ligaments, which play a major role in the function of the knee joint.

Biological reconstruction has been sought to overcome the problems of artificial materials and to enable tendons and ligaments to be attached to bone fragments. Such biological reconstruction uses materials such as allogeneic bone $[6,15]$, distracted autologous bone [7, 16], fibular bone [2, 17, 18], and treated autologous bone [19-21].

Massive bone allografts are still the mainstream of biological reconstruction $[6,15]$. Aponte-Tinao reported long-term results of allograft reconstruction in 35 osteosarcomas of the knee [15]. In this series, 18 patients achieved normal knee function; however, complications treated with additional surgical procedures were recorded for 19 patients, including fractures and three non-unions. These are also seen, although less frequently, with frozen autografts; we experienced only three fractures and two non-unions in the present series. This is because autogenous proteins, growth factors, and cytokines are preserved in the frozen bone [22], which could induce good osteoinduction and osteoconduction to achieve satisfactory bone union and revitalization without eliciting an immune reaction. In addition, there is a perfect fit enabling easy and rigid fixation using simple osteosynthesis techniques and avoiding mal- or non-union. For these reasons, early ROM training is possible, which is very favourable for postoperative knee function. In our series, apart from the one patient who underwent amputation, all patients could bend their knee through $>90^{\circ}$ of flexion and almost half achieved full ROM during the follow-up period. To achieve lower rates of infection, fracture, and non-union of allografts, the use of the Capanna technique (which consists of combined massive allograft and intramedullary vascularized fibula transfer) has been proposed [17]. Houdek et al. reported 18 paediatric patients who underwent lower extremity limb salvage with the use of the Capanna technique, with 17 achieving good or excellent function [18]. However, six patients underwent additional procedures to treat asymptomatic non-union, and this technique seems to be more invasive than the freezing method, considering the fibular resection of the unaffected limb. In addition, we confirmed that bone strength was maintained after treatment with the freezing technique [9], and we consider the frozen autograft itself to be tolerable for normal activity.

A distracted autologous bone and vascularized fibular autograft are useful alternatives to allografts. Physeal distraction followed by bone grafting has been described as a useful tool in the armamentarium to treat children with malignant tumours in close proximity to an open physis, and good postoperative knee function has been reported [16]. However, the limitations of this method are that it is only indicated for young patients and requires several surgical procedures. We have reported intraepiphyseal excision and biological reconstruction with distraction osteogenesis for osteosarcoma around the knee joint $[4,7]$. However, distraction osteogenesis requires a long time to treat with an external fixator and sometimes affects joint function; therefore, we only used it when the tumour-bearing bone was too fragile for freezing or reimplantation. A vascularized fibular autograft is a useful alternative for intercalary defects [2], but it cannot be used alone to provide secure reconstruction of the femur or tibia, and most reports refer to the need for combination with other grafts [17, 18]. In addition, this procedure is technically demanding, and there is a possibility of non-union or bone necrosis, which could prolong the treatment and delay post-operative rehabilitation, affecting postoperative knee function.

After some form of treatment to kill tumour cells, such as autoclaving [19], pasteurization [20], or irradiation [21], recycled bone is useful because it is perfectly matched to the defect, enabling multiple choices for reconstruction and bone excision to maintain knee functions. Several reports have shown good union rates and satisfactory post-operative knee function using biological reconstruction [19-21], although complications such as non-union, fracture, and infection have also been noted. We histologically examined a specimen of autoclaved bone retrieved 24 months post-operatively, in which most of the graft had not incorporated [23]. Conversely, histological examination of a frozen bone showed a small area with active osteocytes and osteoblasts five months after reconstruction [24]. In bone retrieved more than a year after implantation, osteocytes and osteoblasts were observed in broad portions of the frozen autograft, and the cortical host-graft junction showed incorporation along with continuity of bone trabeculae, indicating the onset of osteogenesis in the frozen autograft at an early stage. It may be the case that, as mentioned above, frozen bone preserves bone morphogenetic protein and its osteoinductive ability better than hyperthermic treatments such as autoclaving and pasteurization [22]. Furthermore, if the pedicle-freezing procedure method, which is a specific procedure of frozen autografts and distinguishes from other recycled bone, is possible, the number of osteotomy sites can be decreased, allowing for a faster bone union [25]. In addition, these biological 
reconstruction techniques have issues, such as requiring extracorporeal devices, which make it more likely that the resected bone will be contaminated, whereas the frozen autograft method uses simple surgical techniques without complicated temperature control and special devices. As a result, only two cases of infection (one superficial) were seen in the present study, an incidence lower than that reported in other series on recycled bones [19-21]. Moreover, there was no case of infection in the patients treated by pedicle-freezing method. Vascularity and stability are critical factors in avoiding postoperative infection [26]. The pedicle-freezing method is expected to facilitate early vascularity because of the continuity of one side of the host-graft junction, and this might have helped prevent post-operative infection. However, it is not easy to conquer the infection in recycled bone surgery as well as the other biological methods and endoprosthesic surgery. We consider that rigid fixation, less surgical time, or use of recent antibacterial-agents-coated implants $[27,28]$ are desirable in musculoskeletal tumour surgery.

In joint-sparing surgery, it is particularly important to avoid complications of local recurrence and necrosis from the remaining epiphyseal bone. Recent advances in diagnostic imaging techniques, particularly in MRI, have allowed an accurate definition of the metaphyseal spread of a tumour, and there was no recurrence either from the treated bone or from the remaining epiphyseal bone in our series. In the salvage and reconstruction of the epiphysis, no osteonecrosis of the remaining epiphyseal bone was detected in our series, as with other epiphysis-sparing series [15-17, 20, 21]. However, the minimum follow-up period was two years; patients should be followed up over a longer period to check for late recurrence or future osteoarthritic changes.

This study had some limitations. Pathological evaluations of response to neoadjuvant chemotherapy in case presentations were based on only a part of the tumour: curettage cancellous bone and/or extraskeletal mass; however, we have reported that the histological evaluations of small samples were correlated with the response to chemotherapy and the prognosis [29]. Secondly, comparison between two methods: the free-freezing method and pedicle-freezing method, was not enough to clarify the usefulness of the pedicle-freezing method in terms of bone union or complication of fractures because the patient numbers of each methods were small. Last, this study was a single-arm retrospective study conducted with a small number of patients from a single institute and further study with more patients should be performed.

\section{Conclusion}

Postoperative function after joint-preservation reconstruction using tumour-bearing massive autografts frozen in liquid nitrogen was excellent in 15 of our 18 patients. This procedure provided excellent function for patients with osteosarcoma around the knee, and it may be considered as one of the most useful biological reconstruction methodologies.

\section{Compliance with ethical standards}

Conflict of interest The authors declare that they have no conflict of interest.

Funding There is no funding source.

Ethical approval This is retrospective study. For this type of study formal consent is not required.

Open Access This article is distributed under the terms of the Creative Commons Attribution 4.0 International License (http:// creativecommons.org/licenses/by/4.0/), which permits unrestricted use, distribution, and reproduction in any medium, provided you give appropriate credit to the original author(s) and the source, provide a link to the Creative Commons license, and indicate if changes were made.

\section{References}

1. Manfrini M, Gasbarrini A, Malaguti C, Ceruso M, Innocenti M, Bini S, Capanna R, Campanacci M (1999) Intraepiphyseal resection of the proximal tibia and its impact on lower limb growth. Clin Orthop Relat Res 358:111-119

2. Wong KC, Kumta SM, Antonio GE, Tse LF (2008) Image fusion for computer-assisted bone tumor surgery. Clin Orthop Relat Res 466:2533-2541

3. Chen WM, Wu PK, Chen CF, Chung LH, Liu CL, Chen TH (2012) High-grade osteosarcoma treated with hemicortical resection and biological reconstruction. J Surg Oncol 105:825-829

4. Tsuchiya H, Abdel-Wanis M, Kitano S, Sakurakichi K, Yamashiro T, Tomita K (2002) The natural limb is best: joint preservation and reconstruction by distraction osteogenesis for high-grade juxta-articular osteosarcomas. Anticancer Res 22:2373-2376

5. Yoshida Y, Osaka S, Tokuhashi Y (2010) Analysis of limb function after various reconstruction methods according to tumor location following resection of pediatric malignant bone tumors. World $\mathrm{J}$ Surg Oncol 8:39

6. Kumta SM, Chow TC, Griffith J, Li CK, Kew J, Leung PC (1999) Classifying the location of osteosarcoma with reference to the epiphyseal plate helps determine the optimal skeletal resection in limb salvage procedures. Arch Orthop Trauma Surg 119:327-331

7. Tsuchiya H, Abdel-Wanis ME, Sakurakichi K, Yamashiro T, Tomita K (2002) Osteosarcoma around the knee. Intraepiphyseal excision and biological reconstruction with distraction osteogenesis. J Bone Joint Surg (Br) 84:1162-1166

8. Tsuchiya H, Wan SL, Sakayama K, Yamamoto N, Nishida H, Tomita K (2005) Reconstruction using an autograft containing tumour treated by liquid nitrogen. J Bone Joint Surg (Br) 87:218-225

9. Yamamoto N, Tsuchiya H, Tomita K (2003) Effects of liquid nitrogen treatment on the proliferation of osteosarcoma and the biomechanical properties of normal bone. J Orthop Sci 8:374-380

10. Tsuchiya H, Tomita K, Mori Y, Asada N, Yamamoto N (1999) Marginal excision for osteosarcoma with caffeine assisted chemotherapy. Clin Orthop Relat Res 358:27-35

11. Tsuchiya H, Nishida H, Srisawat P, Shirai T, Hayashi K, Takeuchi A, Yamamoto N, Tomita K (2010) Pedicle frozen autograft reconstruction in malignant bone tumors. J Orthop Sci 15:340-349 
12. Enneking WF, Dunham W, Gebhardt MC, Malawar M, Pritchard DJ (1993) A system for the functional evaluation of reconstructive procedures after surgical treatment of tumors of the musculoskeletal system. Clin Orthop Relat Res 286:241-246

13. Rosen G, Marcove RC, Caparros B, Nirenberg A, Kosloff C, Huvos AG (1979) Primary osteogenic sarcoma: the rationale for preoperative chemotherapy and delayed surgery. Cancer 43:2163-2177

14. Agarwal M, Puri A, Gulia A, Reddy K (2010) Joint-sparing or physealsparing diaphyseal resections: the challenge of holding small fragments. Clin Orthop Relat Res 468:2924-2932

15. Aponte-Tinao L, Ayerza MA, Muscolo DL, Farfalli GL (2015) Survival, recurrence, and function after epiphyseal preservation and allograft reconstruction in osteosarcoma of the knee. Clin Orthop Relat Res 473:1789-1796

16. Betz M, Dumont CE, Fuchs B, Exner GU (2012) Physeal distraction for joint preservation in malignant metaphyseal bone tumors in children. Clin Orthop Relat Res 470:1749-1754

17. Capanna R, Campanacci DA, Belot N, Beltrami G, Manfrini M, Innocenti M, Ceruso M (2007) A new reconstructive technique for intercalary defects of long bones: the association of massive allograft with vascularized fibular autograft. Long-term results and comparison with alternative techniques. Orthop Clin N Am 38(1): $51-60$

18. Houdek MT, Wagner ER, Stans AA, Shin AY, Bishop AT, Sim FH, Moran SL (2016) What is the outcome of allograft and intramedullary free fibula (Capanna technique) in pediatric and adolescent patients with bone tumors? Clin Orthop Relat Res 474: 660-668

19. Khattak MJ, Umer M, Haroon-ur-Rasheed UM (2006) Autoclaved tumor bone for reconstruction: an alternative in developing countries. Clin Orthop Relat Res 447:138-144

20. Jeon DG, Kim MS, Cho WH, Song WS, Lee SY (2007) Pasteurized autograft for intercalary reconstruction: an alternative to allograft. Clin Orthop Relat Res 456:203-210

21. Kim JD, Lee GW, Chung SH (2011) A reconstruction with extracorporeal irradiated autograft in osteosarcoma around the knee. J Surg Oncol 104(2):187-191
22. Takata M, Sugimoto N, Yamamoto N, Shirai T, Hayashi K, Nishida H, Tanzawa Y, Kimura H, Miwa S, Takeuchi A, Tsuchiya H (2011) Activity of bone morphogenetic protein-7 after treatment at various temperatures: freezing vs. pasteurization vs. allograft. Cryobiology 63:235-239

23. Yamamoto N, Tsuchiya H, Nojima T, Sumiya H, Tomita K (2003) Histological and radiological analysis of autoclaved bone 2 years after extirpation. J Orthop Sci 8(1):16-19

24. Tanzawa Y, Tsuchiya H, Shirai T, Hayashi K, Yo Z, Tomita K (2009) Histological examination of frozen autograft treated by liquid nitrogen removed after implantation. J Orthop Sci 14:761-768

25. Shimozaki S, Yamamoto N, Shirai T, Nishida H, Hayashi K, Tanzawa Y, Kimura H, Takeuchi A, Igarashi K, Inatani H, Kato T, Tsuchiya H (2014) Pedicle versus free frozen autograft for reconstruction in malignant bone and soft tissue tumors of the lower extremities. J Orthop Sci 19(1):156-163

26. Friedrich B, Klaue P (1977) Mechanical stability and post-traumatic osteitis: an experimental evaluation of the relation between infection of bone and internal fixation. Injury 9:23-29

27. Hardes J, von Eiff C, Streitbuerger A, Balke M, Budny T, Henrichs MP, Hauschild G, Ahrens H (2010) Reduction of periprosthetic infection with silver-coated megaprostheses in patients with bone sarcoma. J Surg Oncol 101(5):389-395

28. Shirai T, Tsuchiya H, Terauchi R, Tsuchida S, Mizoshiri N, Igarashi K, Miwa S, Takeuchi A, Kimura H, Hayashi K, Yamamoto N, Kubo T (2016) The outcomes of reconstruction using frozen autograft combined with iodine-coated implants for malignant bone tumors: compared with non-coated implants. Jpn J Clin Oncol 46(8):735-740

29. Miwa S, Takeuchi A, Ikeda H, Shirai T, Yamamoto N, Nishida H, Hayashi K, Tanzawa Y, Kimura H, Igarashi K, Tsuchiya H (2013) Prognostic value of histological response to chemotherapy in osteosarcoma patients receiving tumor-bearing frozen autograft. PLoS One 8(8):e71362

30. Zekry KM, Yamamoto N, Hayashi K et al. (2017) Intercalary frozen autograft for reconstruction of malignant bone and soft tissue tumours. Int Orthop. doi:10.1007/s00264-017-3446-x 